\title{
DISCUSSION AFTER PAPER BY O'DELL
}

Ostriker to O'Dell: I do not comprehend how, in the non-burning models for central stars, the addition of a small (non-burning) envelope can alter either the luminosity or the duration significantly. I would have thought that only the radius and hence the temperature would be altered. Thus, discrepancies between the calculated and observed durations may remain a serious problem for the non-burning models. Is my understanding correct?

O'Dell: This is a feature of the work of Salpeter which, I must confess, I do not fully understand.

Paczyński: Was the hydrogen shell ignited?

O'Dell: I think not.

Sugimoto: When we add the hydrogen-rich envelope, the star moves rightwards in the HR diagram, keeping its luminosity constant. As I understand it, this is the reason why the luminosity seems to increase if we compare the two evolutionary tracks at a given effective temperature.

Beaudet: Adding a thin hydrogen shell on top of an homogeneous carbon star does not change the total luminosity but only the effective temperature. It moves the whole track to the right in a HR diagram. Of course, if one adds too much hydrogen, then hydrogen will burn and the above mentioned scheme does not work.

Friedjung: Would determination of the gravitational red shift of the central star be sensitive to asymmetries of the nebula?

O'Dell: I think not, but the problem would be resolved by studying the velocities of different parts of the nebula.

Schwarzschild to O'Dell: Would we commit a very great sin against the observational evidence if we did not consider the 'Harman-Seaton Track' as an evolutionary track but as a patch through which a whole variety of tracks evolve? Could we consider the right hand edge as determined by the condition of the nucleus becoming blue enough to illuminate the nebula and the left hand edge by the condition that the nebula is becoming too dispersed rather than that the nucleus is turning down in luminosity?

O'Dell: I do not think that it is correct to apply such an interpretation. If you look at the average radii of shells about stars of different luminosity, it seems that, even if there are many paths, the general features of the track are clear.

Schwarzschild: If one relaxes the assumption of all nebular stars having the same mass, could you not shift the lower end appreciably? 
O'Dell: I think not as we get the same features by studying both nearby nebulae and others for which we can determine distance and luminosity.

Tayler to O'Dell: The observed density of stars in the diagram which you showed does not agree with the timescale of evolution. Is this due to observational selection?

O'Dell: Yes. 